\title{
Effect of resonant helical magnetic fields on plasma rotation
}

\author{
Q. Yu ${ }^{1}$, S. Günter ${ }^{1}$ and K. H. Finken ${ }^{2}$ \\ ${ }^{1}$ Max-Planck-Institut für Plasmaphysik, EURATOM Association, 85748 Garching, Germany \\ ${ }^{2}$ Institute of Energy Research - Plasma Physics, Forschungszentrum Jülich GmbH, Association \\ EURATOM-FZJ, Trilateral Euregio Cluster, 52425 Jülich, Germany
}

The effect of a resonant helical magnetic field on plasma rotation is investigated numerically based on the two fluid equations. It is found that, depending on the frequency and the direction of the original plasma rotation, a static helical field of a small amplitude can either increase or decrease the rotation speed. With increasing the field amplitude, the plasma rotation frequency approaches the electron diamagnetic drift frequency but rotates in the ion drift direction. These results provide a new understanding of the recent experimental observations of TEXTOR [K.H. Finken et al., Phys. Rev. Letts, 94, 015003(2005)].

PACS: 52.35.Py, 52.35.Vd, 52.55.Tn, 52.35.-g 


\section{Introduction}

The effect of resonant helical magnetic fields on plasma confinement is of great interest to plasma physics research. Three subtopics are of particular concern for a fusion reactor:

(a) Helical field penetration: For the plasma being originally stable to tearing modes, an applied resonant helical field (or error fields of experimental devices) can penetrate through the resonant surface and generate a magnetic island there [1-9]. Recently it was shown on TEXTOR that the relative frequency between the mode and the helical field is important in determining the field penetration [5,6], being in agreement with theoretical results [7-9].

(b) Mode locking: The locking of large magnetic islands by error fields is often observed in experiments, leading to severe confinement degradation in tokamak plasmas or even to disruptions $[1,10,11]$. The mode locking threshold is predicted to be much lower in a fusion reactor than in existing tokamaks due to the stronger magnetic field and lower plasma rotation speed [12].

(c) Edge localized modes (ELMs) mitigation: Helical fields were found to be able to control ELMs while maintaining the pedestal and the confinement of H-mode plasmas, being planned to be applied in ITER [13].

One puzzle arising from the experimental results is the effect of a helical field on plasma rotation. Increased bulk plasma rotation due to an applied static helical field before field penetration was observed on TEXTOR [6]. The tentative explanation for this phenomenon was the induced radial electric field in a stochastic magnetic field generated by the helical field, which leads to the same rotation direction as seen in the experiments [6]. This explanation, however, faces the problem that there is little field ergodicity before field penetration. A change of the bulk plasma rotation from the electron diamagnetic drift direction to the ion's direction by a static penetrated helical field was also observed in experiments [1], a phenomenon not well understood yet. In the framework of reduced MHD equations the static field is known to slow down the bulk plasma rotation $[1,7,10]$. 
Recently, two fluids equations were utilized to study helical field penetration $[8,9,14]$, including the linear study on the effect of a rotating helical field on plasma rotation [9]. As plasma rotation is very important for, e.g., the stability of the resistive wall modes, a better understanding of the experimental results given in Ref. 1 and 6 is desirable. In this paper the effect of a static helical field on plasma rotation is investigated numerically using the (reduced) nonlinear two fluid equations. The obtained results show the same features as observed in the experiments $[1,6]$.

\section{Computational model}

The large aspect-ratio tokamak approximation is utilized. The magnetic field is defined as $\mathbf{B}=\mathrm{B}_{0 \mathrm{t}} \mathbf{e}_{\mathrm{t}}-\left(\mathrm{k}_{\mathrm{t}} / \mathrm{k}_{\theta}\right) \mathrm{B}_{0 \mathrm{t}} \mathbf{e}_{\theta}+\nabla \psi \times \mathbf{e}_{\mathrm{t}}$, where $\psi$ is the helical flux function, $\mathrm{k}_{\theta}=\mathrm{m} / \mathrm{r}$ and $\mathrm{k}_{\mathrm{t}}=\mathrm{n} / \mathrm{R}$ are the wave vector in $\mathbf{e}_{\theta}$ (poloidal) and $\mathbf{e}_{\mathrm{t}}$ (toroidal) direction, $\mathrm{r}$ and $\mathrm{R}$ are the minor and the major radius, $\mathrm{m}$ and $\mathrm{n}$ are the poloidal and toroidal mode numbers of the helical field, and the subscript 0 denotes an equilibrium quantity. The plasma velocity is given by $\mathbf{v}=\mathrm{v}_{\|} \mathbf{e}_{\|}+\nabla \phi \times \mathbf{e}_{\mathrm{t}}$, where $\phi$ is the stream function.

The two fluid equations utilized here include the generalized Ohm's law, the equation of motion in the perpendicular (after taking $\nabla \times$ ) and the parallel (to magnetic field) direction, and the energy and the mass conservation equations. Normalizing the length to the minor radius a, the time $\mathrm{t}$ to the resistive time $\tau_{\mathrm{R}}=\mathrm{a}^{2} \mu_{0} / \eta$, the helical flux $\psi$ to $\mathrm{aB}_{0 t}, \mathbf{v}$ to $\mathrm{a} / \tau_{\mathrm{R}}$, and the electron temperature $\mathrm{T}_{\mathrm{e}}$ and density $\mathrm{n}_{\mathrm{e}}$ to their values at the magnetic axis, theses equations become

$$
\begin{aligned}
& \frac{d \psi}{d t}=E-\eta j+\Omega\left(\nabla_{\|} n_{e}+\nabla_{\|} T_{e}\right) \\
& \frac{d U}{d t}=-S^{2} \nabla_{\|} j+\mu \nabla_{\perp}^{2} U+S_{m} \\
& \frac{d v_{\|}}{d t}=-c_{s}^{2} \nabla_{\|} P / n_{e}+\mu \nabla_{\perp}^{2} v_{\|}
\end{aligned}
$$




$$
\begin{aligned}
& \frac{3}{2} n_{e} \frac{d T_{e}}{d t}=0.78(\Omega / \beta) \nabla_{\|} j+T_{e} n_{e} \nabla_{\|} v_{\|}+n_{e} \nabla_{\|}\left(\chi_{\|} \nabla_{\|} T_{e}\right)+n_{e} \nabla_{\perp}\left(\chi_{\perp} \nabla_{\perp} T_{e}\right)+S_{p} \\
& \frac{d n_{e}}{d t}=(\Omega / \beta) \nabla_{\|} j-\nabla_{\|}\left(n_{e} v_{\|}\right)+\nabla_{\perp}\left(D_{\perp} \nabla_{\perp} n_{e}\right)+S_{n}
\end{aligned}
$$

where $\mathrm{d} / \mathrm{dt}=\partial / \partial \mathrm{t}+\mathbf{v}_{\perp} \cdot \nabla_{\perp}, \quad \mathrm{j}=\nabla_{\perp}^{2} \psi-2 \mathrm{k}_{\mathrm{t}} \mathrm{B}_{0 t} / \mathrm{mR}$ being the toroidal plasma current density, $\eta$ is the normalized resistivity, $E$ is the equilibrium electric field, $\Omega=\beta d_{1}, \beta=4 \pi p_{e} / B^{2}, p_{e}=n_{e} T_{e}, d_{1}=\omega_{c e} / \nu_{e}$, and $\omega_{\text {ce }}$ and $\nu_{\mathrm{e}}$ are the electron cyclotron and the collisional frequency. $S=\tau_{\mathrm{R}} / \tau_{\mathrm{A}}$, where $\tau_{\mathrm{A}}=\mathrm{a} / \mathrm{V}_{\mathrm{A}}$ being the toroidal Alfven time. $U=-\nabla_{\perp}^{2} \phi$ is the plasma vorticity, $c_{s}=\left(T_{e} / m_{i}\right)^{1 / 2}, \mu$ the plasma viscosity, and $\chi_{\|}, \chi_{\perp}$ and $D_{\perp}$ are the parallel and perpendicular heat and particle diffusivity. $S_{p}$ is the heating power, $S_{n}$ is the particle source, and $S_{m}$ is the poloidal momentum source given by

$$
\mathrm{S}_{\mathrm{m}}=\Omega_{\mathrm{E}}\left[1-(\mathrm{r} / \mathrm{a})^{2}\right]^{3},
$$

which leads to an equilibrium poloidal plasma rotation. Cold ion assumption is made here since the ion temperature is significantly lower than the electron's in the considered experiments $[5,6]$.

\section{Numerical Results}

Equations (1)-(5) are solved simultaneously using the initial value code TM1, which has been used for modelling drift tearing modes and neoclassical tearing modes [15-16]. Dedicated numerical methods are utilized in the code to reduce the numerical error $[17,18]$.

The calculations are performed for a single helicity perturbation with $m / n=2 / 1$. The effect of the helical field is taken into account by the boundary condition

$$
\psi_{\mathrm{m} / \mathrm{n}}(\mathrm{r}=\mathrm{a})=\psi_{\mathrm{a}} \cos (\mathrm{m} \theta+\mathrm{n} \phi)
$$

where $\psi_{\mathrm{a}}$ describes the helical magnetic flux amplitude of the $\mathrm{m} / \mathrm{n}$ component at $\mathrm{r}=\mathrm{a}$.

The input parameters are based on TEXTOR experimental parameters. The toroidal magnetic field is $B_{0 t}=2.5 T$, and the plasma minor and major radius are $a=0.47 \mathrm{~m}$ and $\mathrm{R}=1.75 \mathrm{~m}$, respectively. The equilibrium electron temperature and density profiles are modeled by $T_{e}=1.8[1-$ 
$\left.(\mathrm{r} / \mathrm{a})^{2}\right]^{1.2}+0.3(\mathrm{keV})$, and $\mathrm{n}_{\mathrm{e}}=3 \cdot 2\left[1-(\mathrm{r} / \mathrm{a})^{2}\right]^{1.5}+0.3\left(10^{19} \mathrm{~m}^{-3}\right) \quad[5,6,9]$. These parameters lead to $\mathrm{S}=1.97 \times 10^{8}, \Omega=6.3 \times 10^{4}, \mathrm{c}_{\mathrm{s}}=1.2 \times 10^{7}\left(\mathrm{a} / \tau_{\mathrm{R}}\right), \mathrm{d}_{1}=2.5 \times 10^{7}$, and $\chi_{\|}=1.1 \times 10^{9}\left(\mathrm{a}^{2} / \tau_{\mathrm{R}}\right)$. Here $\chi_{\|}=\mathrm{v}_{\mathrm{Te}} /$ $\mathrm{k}_{\|}$is used for calculating $\chi_{\|}$, and $\mathrm{v}_{\mathrm{Te}}$ is the electron thermal velocity [19]. The precise form of $\chi_{\|}$ for a high temperature plasma is more complex as discussed in Ref. [19].

Assuming the perpendicular heat diffusivity and the plasma viscosity to be at the anomalous transport level $0.5 \mathrm{~m}^{2} / \mathrm{s}$, one finds $\chi_{\perp}=\mu=21\left(\mathrm{a}^{2} / \tau_{\mathrm{R}}\right)$ in normalized units. Furthermore, $\mathrm{D}_{\perp}=\chi_{\perp} / 5$ is taken. These parameters are used as input for our calculations except mentioned elsewhere. A monotonic profile of the safety factor $\mathrm{q}$ is used, and the $\mathrm{q}=2$ rational surface locates at $r_{s}=0.628 \mathrm{a}$ in agreement with the experiments $[5,6,9]$. The $m / n=2 / 1$ tearing mode is stable without an externally applied helical field.

For simplicity, we first study the case with a constant electron temperature. With different initial equilibrium plasma rotation velocities, the effect of a helical field on the plasma rotation is different. The radial profiles of the (normalized) $\mathrm{m} / \mathrm{n}=0 / 0$ component of the poloidal plasma rotation velocity, $V_{\theta}$, in steady state are shown in figure 1 for two cases: (a) $\omega_{0} \equiv \omega_{\mathrm{E} 0} / \omega_{*_{\mathrm{e} 0}}=0.42$ (black curves) with $\psi_{\mathrm{a}}=10^{-5}$ (solid) and 0 (dashed). (b) $\omega_{0}=-0.42$ (red) with $\psi_{\mathrm{a}}=0.4 \times 10^{-5}$ (solid) and $1.14 \times 10^{-5}$ (dotted) and 0 (dashed), where $\omega_{\mathrm{E} 0}$ and $\omega_{*_{\mathrm{e}} 0}$ are the original equilibrium plasma rotation frequency and the electron diamagnetic drift frequency at the rational surface. The $\omega_{0}$ values are also marked near the corresponding curves in the figure. The positive (negative) value of $\omega_{0}$ refers to the rotation in the the ion (electron) diamagnetic drift direction. It is seen that the rotation is speeded up by the static helical field for $\omega_{0}=0.42$, while for $\omega_{0}=-0.42$ the rotation first slows down (for $\psi_{\mathrm{a}}=0.4 \times 10^{-5}$ ) and then changes its direction to the ion diamagnetic drift direction for larger helical field amplitude $\left(\psi_{\mathrm{a}}=1.14 \times 10^{-5}\right)$. These results are consistent with the experimental observations [1,6]. For $\omega_{0}>1$ the plasma rotation velocity is always found to decrease. 
The results shown in Fig. 1 can be understood from the generalized Ohm's law. Assuming the perturbed helical flux $\psi_{1} \sim \exp [i(-\omega t+\operatorname{m} \theta+n \phi)]$, for a sufficiently small $\psi_{\mathrm{a}}$ it is found from Eq. (1) that in the lowest order the toroidal current perturbation at the rational surface $r_{s}$ is given by

$$
\mathrm{j}_{1}\left(\mathrm{r}_{\mathrm{s}}\right)=\mathrm{i}\left(\omega-\omega_{\mathrm{E}}-\omega_{*}\right) \psi_{1} / \eta
$$

where the subscript 1 denotes perturbed quantities, $\omega$ is the mode frequency, $\omega_{\mathrm{E}}=\mathrm{V}_{\theta} \mathrm{m} / \mathrm{r}$ being the frequency due to $\mathrm{V}_{\theta}, \omega_{*_{\mathrm{e}}}=\mathrm{V}_{*_{\mathrm{et}}} \mathrm{n} / \mathrm{R}=\mathrm{V}_{*_{\mathrm{e} \theta}} \mathrm{m} / \mathrm{r}$ being the frequency due to the electron diamagnetic

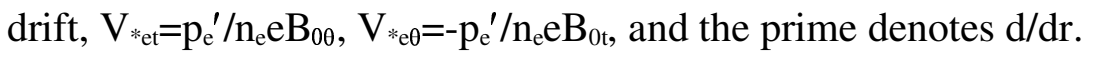

The current perturbation together with the radial magnetic field perturbation, $b_{\mathrm{r} 1}$, leads to a poloidal electromagnetic torque of the $\mathrm{m} / \mathrm{n}=0 / 0$ component, $\mathrm{T}_{0 / 0}=\mathrm{r}\left(\mathrm{j}_{1}{ }^{*} \mathrm{~b}_{\mathrm{r} 1}+\mathrm{j}_{1} \mathrm{~b}_{\mathrm{r} 1}{ }^{*}\right) / 2$, where the superscript * refers to the complex conjugated part. At the rational surface one finds

$$
\mathrm{T}_{0 / 0}\left(\mathrm{r}_{\mathrm{s}}\right)=\mathrm{m}\left(\omega-\omega_{\mathrm{E}}-\omega *_{\mathrm{e}}\right)\left|\psi_{1}\right|^{2} / \eta
$$

As the mode frequency is given by the applied field frequency, for a static helical field $\omega=0$. Eq. (9) is then reduced to

$$
\mathrm{T}_{0 / 0}\left(\mathrm{r}_{\mathrm{s}}\right)=-\mathrm{m} \omega_{\mathrm{E}}\left(1+\omega_{*} / \omega_{\mathrm{E}}\right)\left(\left|\psi_{1}\right|\right)^{2} / \eta
$$

In the MHD limit $\omega_{*_{\mathrm{e}}}=0$ is used, and $\mathrm{T}_{0 / 0}\left(\mathrm{r}_{\mathrm{s}}\right)$ is further simplified to $-\mathrm{m} \omega_{\mathrm{E}}\left(\left|\psi_{1}\right|\right)^{2} / \eta$, which is always opposite to the plasma rotation direction, slowing down the rotation speed, no matter in which direction the plasma originally rotates.

When taking into account the electron diamagnetic drift, the torque direction depends on the rotation frequency and direction. Eq. (10) indicates that for $\left(1+\omega *_{\mathrm{e}} / \omega_{\mathrm{E}}\right)<0 \quad$ (plasma rotates in the ion drift direction with a frequency smaller than $\omega_{*}$ ), the torque is in the rotation direction. For $\left(1+\omega * d \omega_{\mathrm{E}}\right)>0$, however, the torque is in the opposite direction.

The above analysis is consistent with numerical results. Corresponding to Fig. 1, the radial profiles of $\mathrm{T}_{0 / 0}$ in steady state are shown in figure 2 for $\omega_{0}=0.42$ (black curve) and 2.08 (blue) with $\psi_{\mathrm{a}}=0.4 \times 10^{-5}$. The red curves are for $\omega_{0}=-0.42$ with $\psi_{\mathrm{a}}=0.4 \times 10^{-5}$ (solid) and $1.14 \times 10^{-5}$ (dotted). 
The $\omega_{0}$ values are also marked near the corresponding curves in the figure. The torque peaks at the rational surface and is in the plasma rotation direction for $\omega_{0}=0.42$ (see Fig. 1) but is opposite to the rotation direction for $\omega_{0}=2.08$, as expected. For $\omega_{0}=-0.42$ the torque is also directed opposite to the plasma rotation for a small $\psi_{\mathrm{a}}$, and it even changes the rotation direction for $\psi_{\mathrm{a}}=1.14 \times 10^{-5}$. The torque direction is as predicted by Eq. (10) for all cases.

For a small helical field amplitude, the electromagnetic torque is localized around the rational surface and tends to modify the local plasma rotation velocity. The plasma viscosity, however, will response to the local velocity change and tend to keep the neighbouring plasma to rotate together with the local plasma at the rational surface. This results in a more global change in the rotation velocity profile, as seen from Fig. 1, such that the electromagnetic torque is balanced by the viscous torque. With increasing helical field amplitude, the electromagnetic torque becomes larger as seen from Eq. (10), and it will eventually lead to $\omega_{\mathrm{E}} \approx-\omega_{*_{\mathrm{e}}}$ at the rational surface for a static helical field, such that electromagnetic torque is not too large to be balanced by the viscous torque. In this case the global rotation velocity profile also significantly changes.

For a systematic study, the normalized plasma rotation frequency, $\omega_{\mathrm{En}} \equiv-\omega_{\mathrm{E}} / \omega_{* \mathrm{e} 0}(\mathrm{solid}$ curve), and the normalized electron diamagnetic drift frequency, $\omega_{* \mathrm{en}} \equiv \omega \omega_{\mathrm{e}} / \omega_{*_{\mathrm{e} 0}}$ (dotted curve), at the rational surface in steady state are shown as a function of $\psi_{\mathrm{a}}$ in figure 3 for three cases, $\omega_{0}=0.42$ (black curve), 2.08 (blue) and -0.42 (red). The $\omega_{0}$ value for these curves can be also found from the $\omega_{\mathrm{En}}$ value at $\psi_{\mathrm{a}}=0$. The dashed curve shows the normalized island width w/a multiplied by a factor 10 . It is seen that for $\omega_{0}=0.42$, the normalized rotation frequency $\omega_{\text {En }}$ first increases with increasing $\psi_{\mathrm{a}}$ and then decreases following the decrease of $\omega_{* \mathrm{en}}$. For a sufficiently large $\psi_{\mathrm{a}}$ or island width, the plasma density gradient decreases in the island region due to the parallel transport, and therefore $\omega_{*_{e}}$ decreases. The sudden increase in the island width corresponds to the helical field penetration. For $\omega_{0}=2.08$ the normalized frequency $\omega_{\text {En }}$ decreases 
towards $\omega_{* \text { en }}$. With $\omega_{0}=-0.42, \omega_{\text {En }}$ increases from a negative value to approach $\omega_{* \text { en. }}$ No matter what is the initial rotation speed or direction, the nonlinear state $\omega_{\mathrm{En}} \approx \omega_{*_{\mathrm{en}}}$ (or $\omega_{\mathrm{E}} \approx-\omega *_{\mathrm{e}}$, plasma rotates in the ion drift direction) is always reached for a sufficiently large $\psi_{\mathrm{a}}$. In this state the torque is minimum as seen from Eq. (10).

For a non-constant electron temperature, similar results are found as shown in figure 4, in which $\omega_{\text {En }}$ (solid curve) and $\omega_{* \text { en }}\left(\right.$ dotted) are shown as a function of $\psi_{\mathrm{a}}$ for $\omega_{0}=0$. The black (red) curves are for constant (non-constant) electron temperature. The dashed curve shows 10×w/a. The case with a non-constant electron temperature has a larger values of $\omega_{\mathrm{En}}$ and $\omega_{* \text { en }}$ but a smaller value of w/a. A larger $\psi_{\mathrm{a}}$ is required for the field penetration with a non-constant electron temperature, since the frequency difference between the mode and the field is larger due to the contribution of the temperature gradient to $\omega_{*}$. It is seen that a static field forces the plasma to rotate in the ion diamagnetic drift direction, even if originally there is no plasma rotation.

In figure 5 the normalized frequencies $\omega_{\text {En }}$ (solid curve) and $\omega_{* \text { en }}$ (dotted curve) are shown as a function of $\log (\mathrm{S})$ for $\omega_{0}=0$ and $\psi_{\mathrm{a}}=10^{-5}$. The other parameters are the same as those of Fig. 3 . The dashed curve shows 10×w/a. The electromagnetic torque exerted on the island by the helical field is larger for a larger magnetic Renolds number $S$, so that $\omega_{\mathrm{En}}$ increases with increasing $S$, which in turn leads to field penetration at $\mathrm{S}=1.2 \times 10^{8}$ as indicated by a sudden increase in the island width. After field penetration one finds $\omega_{\mathrm{E}} \approx-\omega *_{\mathrm{e}}$ similar to the results shown in Figs. 3 and 4 , and $\omega_{* \mathrm{e}} \approx 0.9 \omega_{*_{\mathrm{e} 0}}$ due to the small island width. Fig. 5 suggests that the plasma rotation will be more easily generated by the helical field for a reactor plasma with a high $\mathrm{S}$ value.

\section{Discussion and Summary}

The above results are obtained for the case of poloidal plasma rotation. In tokamak experiments the plasma rotation is mostly in the toroidal direction due to the neoclassical effect 
not considered here. For toroidal rotation, Eq. (9) is found to be modified as

$$
\mathrm{T}_{0 / 0}\left(\mathrm{r}_{\mathrm{s}}\right)=\mathrm{n}\left(\omega-\omega_{\mathrm{E}}-\omega_{*}\right)\left|\psi_{1}\right|^{2} / \eta,
$$

where $\omega_{\mathrm{E}}=\mathrm{V}_{\mathrm{t}} \mathrm{n} / \mathrm{R}$ being the mode frequency due to the toroidal plasma rotation velocity $\mathrm{V}_{\mathrm{t}}$. The electron diamagnetic drift is in the counter plasma current direction, so that the helical field drives the plasma to rotates in the co-current direction. A larger toroidal rotation speed than the poloidal one will be driven by the helical field, since the toroidal rotation speed should be $(\mathrm{m} / \mathrm{n})\left(\mathrm{R} / \mathrm{r}_{\mathrm{s}}\right)$ times larger than the poloidal one for the same change in the frequency.

Our results indicates that a static resonant magnetic perturbation of a single helicity can drive plasma flow, showing the same features as observed in the experiments $[1,6]$. Future studies using the toroidal rotation and a stochastic magnetic field will be helpful for a further comparison with the experimental results.

For a fusion reactor the plasma rotation velocity is expected to be low, since the neutral beam injection heating will not be present for a burning plasma. A slowly rotating plasma will be more easily subject to plasma instabilities due to the weaker stabilizing effect of the wall. As the diamagnetic drift velocity is very large in the pedestal region of $\mathrm{H}$-mode plasmas, while the local parallel transport is not too fast, a local fast plasma rotation could be generated by a static helical field of an appropriate amplitude, especially when considering the reduced plasma viscosity in the pedestal region. Spontaneous plasma rotation in the co-current direction has been found in $\mathrm{H}$-mode plasmas on several tokamaks [20]. It would be of interest to study whether the intrinsic machine error field is resonant at the plasma edge and large enough to cause such a rotation. In the tokamak core region the parallel transport is much faster, and $\omega *_{\mathrm{e}}$ begins to decrease due to the flattening of the radial electron pressure profile even before field penetration [8]. In this case a rotating helical field would still be able to drive plasma rotation. Eqs. (9) and (11) suggest that a helical field rotating in the ion diamagnetic drift direction can more efficiently drive plasma rotation. This was indeed found to be the case in TEXTOR experimental results [6]. 
In summary, the effect of a resonant helical field on plasma rotation is investigated based on two fluid equations, providing a new insight into previous experimental results. It is found that: (a) Before field penetration the rotation is speeded up by a static helical field in case the original rotation is in the ion diamagnetic drift direction with a frequency smaller than $\omega_{* \text { e }}$, but it is slowed down otherwise. (b) For a sufficiently large field amplitude, the plasma rotation frequency approaches the electron diamagnetic drift frequency but rotates in the ion drift direction. 


\section{References}

[1] T.C. Hender, R. Fitzpatrick, A.W. Morris, P.G. Carolan, R.D. Durst, T. Edlington, J. Ferreira, S.J. Fielding, P.S. Haynes, J. Hugill, I.J. Jenkins, R.J. La Haye, B.J. Parham, D.C. Robinson, T.N. Todd, M. Valovic and G. Vayakis, Nucl. Fusion 32, 2091 (1992).

[2] R.J. Buttery, M. De' Benedetti, T.C. Hender and B.J.D. Tubbing, Nucl. Fusion 40, 807 (2000).

[3] R. J. La Haye, R. Fitzpatrick, T. C. Hender, A. W. Morris, J. T. Scoville, and T. N. Todd, Phys. Fluids B 4, 2098 (1992).

[4] S. M. Wolfe, I. H. Hutchinson, R. S. Granetz, J. Rice, and A. Hubbard, Phys. Plasmas 12, $056110(2005)$.

[5] H.R. Koslowski, Y. Liang, A. Krämer-Flecken, K. Löwenbrück, M. von Hellermann, E. Westerhof, R. C. Wolf, O. Zimmermann and the TEXTOR team, Nucl. Fusion 46, L1 (2006).

[6] K. H. Finken, S. S. Abdullaev, M. F. M. de Bock, M. von Hellermann, M. Jakubowski, R. Jaspers, H. R. Koslowski, A. Krämer-Flecken, M. Lehnen, Y. Liang, A. Nicolai, R. C. Wolf, O. Zimmermann, M. de Baar, G. Bertschinger, W. Biel, S. Brezinsek, C. Busch, A. J. H. Donné, H. G. Esser, E. Farshi, H. Gerhauser, B. Giesen, D. Harting, J. A. Hoekzema, G. M. D. Hogeweij, P. W. Hüttemann, S. Jachmich, K. Jakubowska, D. Kalupin, F. Kelly, Y. Kikuchi, A. Kirschner, R. Koch, M. Korten, A. Kreter, J. Krom, U. Kruezi, A. Lazaros, A. Litnovsky, X. Loozen, N. J. Lopes Cardozo, A. Lyssoivan, O. Marchuk, G. Matsunaga, Ph. Mertens, A. Messiaen, O. Neubauer, N. Noda, V. Philipps, A. Pospieszczyk, D. Reiser, D. Reiter, A. L. Rogister, M. Sakamoto, A. Savtchkov, U. Samm, O. Schmitz, R. P. Schorn, B. Schweer, F. C. Schüller, G. Sergienko, K. H. Spatschek, G. Telesca, M. Tokar, R. Uhlemann, B. Unterberg, G. Van Oost, T. Van Rompuy, G. Van Wassenhove, E. Westerhof, R. Weynants, S. Wiesen, and Y. H. Xu, Phys. Rev. Letts, 94, 015003(2005).

[7] R. Fitzpatrick, Nucl. Fusion 33, 1049 (1993).

[8] Q. Yu, S. Günter, Y. Kikuchi, and K. H. Finken, Nuclear Fusion 48, 024007 (2008). 
[9] Kikuchi, M. F. M. de Bock, K. H. Finken, M. Jakubowski, R. Jaspers, H. R. Koslowski, A. Kraemer-Flecken, M. Lehnen, Y. Liang, G. Matsunaga, D. Reiser, R. C. Wolf, and O. Zimmermann, Phys. Rev. Letts, 97, 085003(2006).

[10] M.F.F. Nave and J.A. Wesson, Nucl. Fusion 30, 2575 (1990).

[11] H. Zohm, A. Kallenbach, H. Bruhns, G. Fussmann, Europhys. Lett. 11, 745 (1990).

[12] Q. Yu and S. Günter, Nucl. Fusion 48, 065004 (2008).

[13] T. E. Evans, R. A. Moyer, P. R. Thomas, J. G. Watkins, T. H. Osborne, J. A. Boedo, E. J. Doyle, M. E. Fenstermacher, K. H. Finken, R. J. Groebner, M. Groth, J. H. Harris, R. J. La Haye, C. J. Lasnier, S. Masuzaki, N. Ohyabu, D. G. Pretty, T. L. Rhodes, H. Reimerdes, D. L. Rudakov, M. J. Schaffer, G. Wang, and L. Zeng, Phys. Rev. Letts, 92, 235003 (2004).

[14] F. L. Waelbroeck, Phys. Plasmas 10, 4040 (2003).

[15] Q. Yu, S. Günter and Bruce D. Scott, Phys. Plasmas 10, 797(2003).

[16] Q. Yu, S. Günter and K. Lackner, Phys. Plasmas 11, 140(2004).

[17] S. Günter, Q. Yu, J. Krüger, and K. Lackner, J. Comp. Phys. 209, 35 (2005)

[18] Q. Yu, Phys. Plasmas 13062310 (2006)

[19] Z. Chang and J.D. Callen, Phys. Fluids B4, 1167(1992).

[20] J.E. Rice, A. Ince-Cushman, J.S. deGrassie, L.-G. Eriksson, Y. Sakamoto, A. Scarabosio, A. Bortolon, K.H. Burrell, B.P. Duval, C. Fenzi-Bonizec, M.J. Greenwald, R.J. Groebner, G.T. Hoang, Y. Koide, E.S. Marmar, A. Pochelon and Y. Podpaly, Nucl. Fusion 47, 1618 (2007). 


\section{CAPTION}

Figure 1 (color online) Radial profiles of the normalized $\mathrm{m} / \mathrm{n}=0 / 0$ component poloidal velocity, $V_{\theta}$, for two cases: (a) $\omega_{0} \equiv-\omega_{\mathrm{E} 0} / \omega_{*_{\mathrm{e} 0}}=0.42$ (black curves) with $\psi_{\mathrm{a}}=10^{-5}$ (solid) and 0 (dashed). (b) $\omega_{0}=-0.42$ (red) with $\psi_{\mathrm{a}}=0.4 \times 10^{-5}$ (solid) and $1.14 \times 10^{-5}$ (dotted) and 0 (dashed). The $\omega_{0}$ values are also marked near the corresponding curves.

Figure 2 (color online) Radial profiles of $\mathrm{T}_{0 / 0}$ in steady state for $\omega_{0}=0.42$ (black curve) and 2.08 (blue) with $\psi_{\mathrm{a}}=0.4 \times 10^{-5}$. The red curves are for $\omega_{0}=-0.42$ with $\psi_{\mathrm{a}}=0.4 \times 10^{-5}$ (solid) and $1.14 \times 10^{-5}$ (dotted). The $\omega_{0}$ values are also marked near the corresponding curves.

Figure 3 (color online) $\omega_{\mathrm{En}} \equiv-\omega_{\mathrm{E}} / \omega_{*_{\mathrm{e} 0}}($ solid curve $), \omega_{*_{\mathrm{en}}} \equiv \omega *_{\mathrm{e}} / \omega_{*_{\mathrm{e} 0}}($ dotted $)$ and $10 \mathrm{w} / \mathrm{a}$ (dashed) versus $\psi_{\mathrm{a}}$ for $\omega_{0}=0.42$ (black), 2.08 (blue) and -0.42 (red). $\omega_{\mathrm{En}} \approx \omega *_{\mathrm{en}}\left(\right.$ or $\omega_{\mathrm{E}} \approx-\omega *_{\mathrm{e}}$ ) for a sufficiently large $\psi_{\mathrm{a}}$. The $\omega_{0}$ value for these curves can be also found from the $\omega_{\mathrm{En}}$ value at $\psi_{\mathrm{a}}=0$.

Figure 4 (color online) $\omega_{\text {En }}$ (solid curve), $\omega *_{\text {en }}$ (dotted) and 10w/a (dashed) versus $\psi_{\text {a }}$ for $\omega_{0}=0$. The black (red) curves are for a constant (non-constant) electron temperature. The case with a non-constant electron temperature has a larger values of $\omega_{\mathrm{En}}$ and $\omega_{* \mathrm{en}}$ but a smaller value of w/a. A static field drives the plasma rotation in the ion diamagnetic drift direction.

Figure 5 (color online) $\omega_{\mathrm{En}}$ (solid curve), $\omega_{* \text { en }}$ (dotted) and $10 \mathrm{w} / \mathrm{a}$ (dashed) versus $\log (\mathrm{S})$ for $\psi_{\mathrm{a}}=10^{-5}$ and $\omega_{0}=0 . \omega_{\mathrm{En}}$ increases with increasing S. After field penetration $\omega_{\mathrm{E}} \approx-\omega_{* \mathrm{e}}$. 


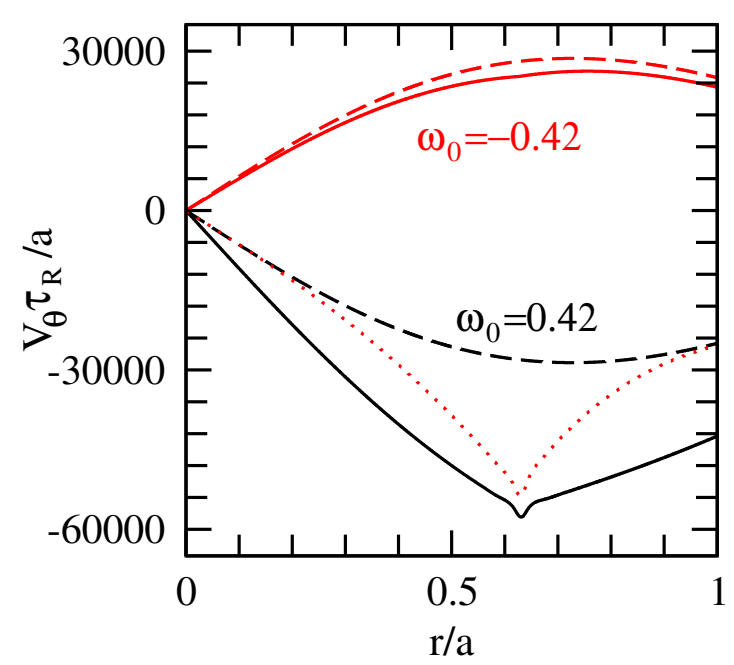

Figure 1 


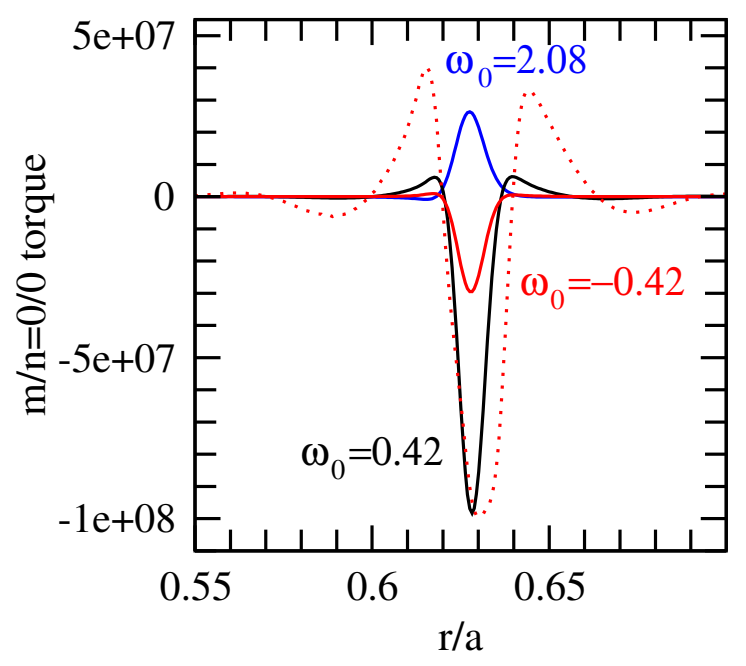

Figure 2 


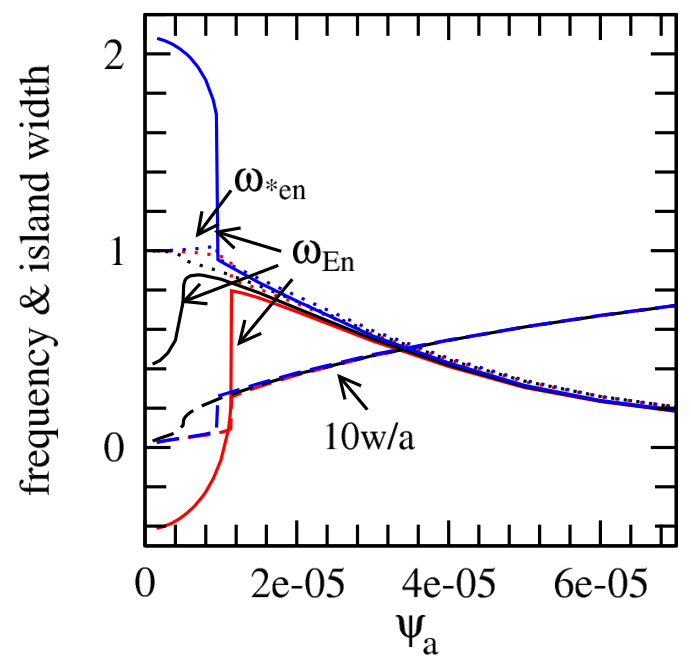

Figure 3 


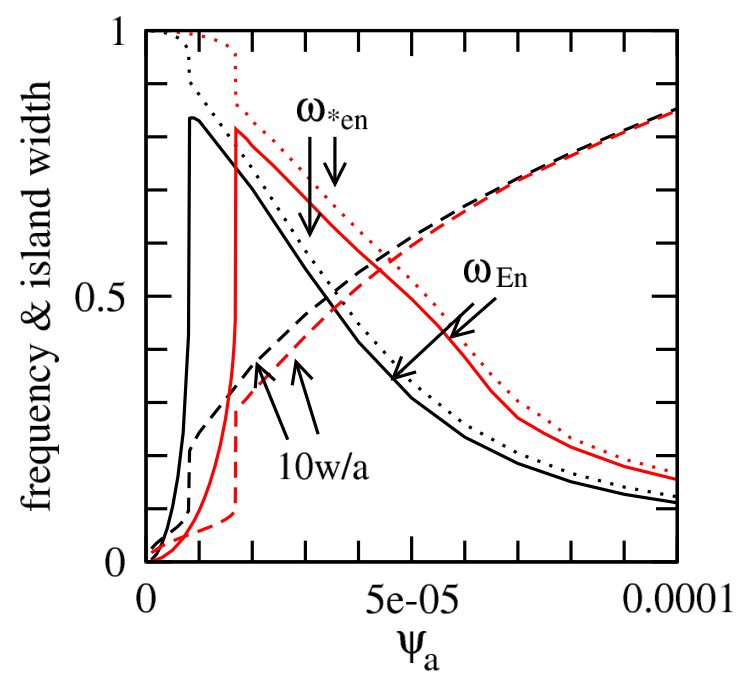

Figure 4 


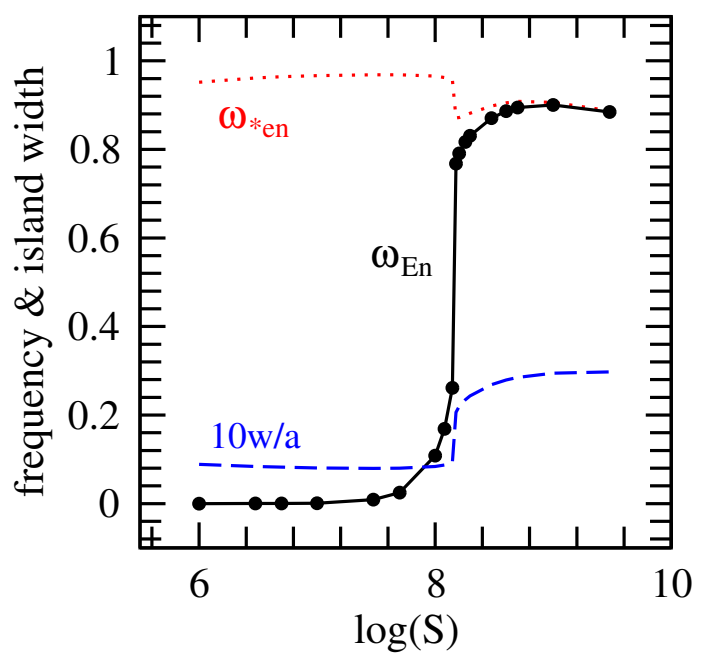

Figure 5 